\title{
Nonlinear vortex development in rotating flows
}

Hewitt, R.E. and Mullin, T. and Tavener, S.J. and Khan, M.A.I. and Treacher, P.D.

2008

MIMS EPrint: 2012.21

Manchester Institute for Mathematical Sciences

School of Mathematics

The University of Manchester

\footnotetext{
Reports available from: http://eprints.maths.manchester.ac.uk/

And by contacting: The MIMS Secretary

School of Mathematics

The University of Manchester

Manchester, M13 9PL, UK
} 


\title{
PHILOSOPHICAL TRANSACTIONS

\section{Nonlinear vortex development in rotating flows}

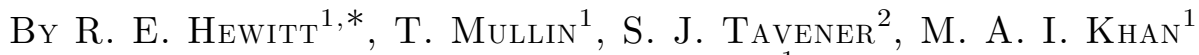 \\ AND P. D. TREACHER ${ }^{1}$ \\ ${ }^{1}$ Manchester Centre for Nonlinear Dynamics, University of Manchester, \\ Oxford Road, Manchester M13 9PL, UK \\ ${ }^{2}$ Department of Mathematics, Colorado State University, Fort Collins, \\ CO 80523, USA
}

We present the results of a combined experimental and numerical investigation into steady secondary vortex flows confined between two concentric right circular cylinders. When the flow is driven by the symmetric rotation of both end walls and the inner cylinder, toroidal vortex structures arise through the creation of stagnation points (in the meridional plane) at the inner bounding cylinder or on the mid-plane of symmetry. A detailed description of the flow regimes is presented, suggesting that a cascade of such vortices can be created. Experimental results are reported, which visualize some of the new states and confirm the prediction that they are stable to (mid-plane) symmetrybreaking perturbations.

We also present some brief results for the flows driven by the rotation of a single end wall. Vortex structures may also be observed at low Reynolds numbers in this geometry. We show that standard flow visualization methods lead to some interesting nonaxisymmetric particle paths in this case.

Keywords: driven cavity flow; rotating flows; disc flows

\section{Introduction}

The steady flow within a single circular cylinder, with one or both end walls driving the fluid within the cavity, has been the subject of a great deal of numerical and experimental research. This geometry has proved to be a useful tool for studying the development of secondary structures on a forced primary vortex flow. Previous studies have shown that a rapid development of a vortex 'bubble' ${ }^{1}$ may occur on the primary vortex axis (see Vogel 1968; Escudier 1984).

Vogel (1968) was the first to study the flow driven by a single rotating end wall. He showed that it was possible to form a pair of stagnation points midway along the central core of the driven columnar vortex (the primary flow state)

* Author for correspondence (richard.e.hewitt@manchester.ac.uk).

${ }^{1}$ In the associated literature, the phrase 'bubble' has been used to indicate a closed region of recirculation in the meridional plane.

One contribution of 6 to a Theme Issue 'Experimental nonlinear dynamics II. Fluids'. 
above a certain range of Reynolds numbers. Vogel suggested that the creation of a local secondary (axisymmetric) recirculation by this flow feature was a steady form of the ill-defined 'vortex breakdown' phenomenon.

Driven cavity flows of the type described here are important both scientifically and technologically. Indeed, there are few flows of similar practical significance in which the boundary conditions are so unambiguously defined and, as a consequence, offer an ideal framework in which meaningful and detailed comparisons can be made between the results of experiments and numerical computations. The flow configuration has a wide range of applicability including, for example, swirl combustion chambers and the flow between enclosed, rotating and data storage media. In such cases, a deeper understanding of the formation and location of vortex bubbles and their associated stagnation points is vital in gaining further insight into the origins of more complicated dynamics.

Escudier (1984) extended Vogel's work with a single driving boundary and presented the results of a systematic sequence of experiments. He was able to establish the parameter ranges for the existence of steady secondary vortices. He also showed that the vortices do not form in domains with an aspect ratio smaller than a critical value and that multiple steady vortices (aligned on the rotation axis) and temporal dependence can be found at sufficiently large Reynolds numbers and aspect ratios.

In the work of Escudier, the appearance of the vortex bubbles was discussed in the vocabulary of stability theory, with parameter space diagrams (spanned by the aspect ratio and rotational Reynolds number) showing 'stability boundaries' for 'breakdown'. However, Tsiverblit (1993) established that the appearance of the single secondary vortex is not caused by any hydrodynamic instability or bifurcation but is the smooth evolution of one steady axisymmetric flow to another as the Reynolds number is increased in an appropriate control parameter regime. That this flow feature is not induced by an instability means that there is no (local) underlying solution to the Navier-Stokes system in this geometry, for which the flow does not develop a closed recirculation region in the meridional flow.

In contrast to the work of Escudier (1984), a more recent paper by Mullin et al. (1998) gave numerical evidence for the development of off-axis toroidal vortices in a small aspect ratio driven cavity. In these flows, unlike those described by Escudier, a vortex was suggested to develop in the main bulk of the flow, rather than at the rotation axis or an inner boundary. Their work was inspired by the work of Valentine \& Jahnke (1994) on symmetrically driven flows.

The primary aim in this investigation is to address the stability of the newly predicted 'off-axis' toroidal vortices and whether they can be observed in a laboratory experiment. We also consider the nonlinear development for increasing Reynolds numbers both numerically and experimentally. The appearance of multiple regions of recirculation in the meridional plane is not purely a finite Reynolds number phenomenon; toroidal vortices may also develop at low Reynolds numbers. In particular, an analysis of the slow-flow regime by Hills (2001) has shown that a cascade of toroidal vortices can be found at large aspect ratios. In this work, we briefly comment on some new features observed in the experimental visualization of such lid-driven cavity flows.

The presentation of material in this paper is as follows. In $\S 2$ we formulate the problem and in $\S 3$ we describe the experimental configuration. In $\S 4$ we present 
(a)

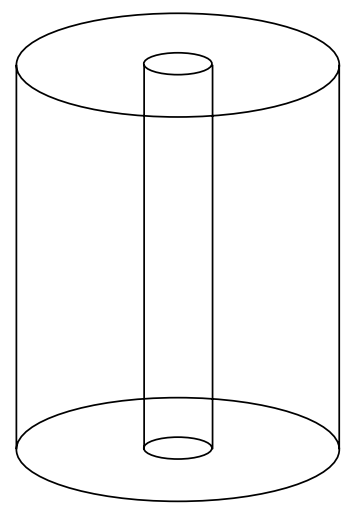

(b)

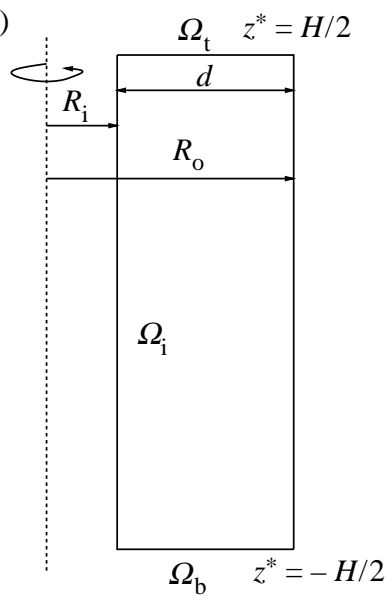

Figure 1. (a) The flow geometry. (b) A (half) meridional view of the flow domain. The top $\left(z^{*}=H / 2\right)$, bottom $\left(z^{*}=-H / 2\right)$ and inner $\left(r^{*}=R_{\mathrm{i}}\right)$ boundaries are set to rotate with angular frequencies $\Omega_{\mathrm{t}, \mathrm{b}, \mathrm{i}}$ while the outer cylinder $\left(r^{*}=R_{\mathrm{o}}\right)$ is stationary.

both numerical and experimental results and make comparisons between the two using flow visualization techniques. Finally, $\$ 5$ provides a general discussion of the implications and future extensions of the work.

\section{Formulation}

We consider the flow domain shown schematically in figure 1 . The annular region contains a viscous, incompressible, Newtonian fluid of kinematic viscosity $\nu$ and density $\rho$. The experimental configuration (described below) allows for two driving mechanisms for the flow: (i) $\Omega_{\mathrm{t}}=\Omega_{\mathrm{i}}=\Omega_{\mathrm{b}}=\Omega$ or (ii) $\Omega_{\mathrm{b}}=\Omega$ and $\Omega_{\mathrm{i}}=\Omega_{\mathrm{t}}=0$. Here, $\Omega_{\mathrm{t}, \mathrm{b}, \mathrm{i}}$ indicates the angular frequency of the top/bottom/inner bounding walls, respectively. The outer wall is always stationary.

We assume that the flow remains axisymmetric and describe it with respect to a cylindrical polar coordinate system $\left(r^{*}, \theta, z^{*}\right)$, in which the corresponding velocity components are $\left(u^{*}, v^{*}, w^{*}\right)$. This coordinate system is centred on the intersection of the rotation axis and the mid-plane of symmetry.

We non-dimensionalize using the gap width $d=R_{\mathrm{o}}-R_{\mathrm{i}}$ as the length scale and $\Omega R_{\mathrm{i}}$ as the velocity scale. Furthermore, for our computational solutions, we shall rescale the flow domain to a unit square, thereby introducing an aspect ratio parameter into the problem and a radius ratio. We therefore define $r^{*}=R_{\mathrm{i}}+d r$ and $z^{*}=d z \Gamma$, where

$$
\Gamma=H / d>0, \quad \eta=R_{\mathrm{i}} / R_{\mathrm{o}}<1
$$

and $(r, z) \in[0,1] \times[-1 / 2,1 / 2]$. The governing Navier-Stokes equations in this form then possess a Reynolds number defined by

$$
R e=\frac{\Omega R_{\mathrm{i}} d}{\nu} .
$$


Table 1. Velocity convergence data, measured relative to the analytical solution for circular Couette flow, with $\eta=0.1$ and $R e=10$. (The flow domain is spanned by $N_{x} \times N_{y}$ elements and $\Delta v$ is the absolute difference between the analytical solution and the numerical result measured at the central point $r=0.5, z=0$. Tolerance of the numerical method is $10^{-8}$.)

\begin{tabular}{llr}
\hline$N_{x} \times N_{y}$ & $\Delta v$ & ratio \\
\hline $4 \times 4$ & $6.10683 \times 10^{-4}$ & - \\
$8 \times 8$ & $1.19152 \times 10^{-4}$ & 5.13 \\
$16 \times 16$ & $8.00082 \times 10^{-6}$ & 14.90 \\
$32 \times 32$ & $5.15248 \times 10^{-7}$ & 15.53 \\
\hline
\end{tabular}

The only non-trivial boundary conditions are for the swirl component of the velocity field, $v^{*}=\Omega R_{\mathrm{i}} v(r, z)$, which can be non-dimensionalized as

$$
v=\Omega_{\mathrm{i}} / \Omega \quad \text { on } r=0
$$

and

$$
v=\frac{\Omega_{\mathrm{t}, \mathrm{b}}}{\Omega}\left(1+\frac{1-\eta}{\eta} r\right) \quad \text { on } z= \pm \frac{1}{2} .
$$

As noted above, the combinations $\Omega_{\mathrm{t}, \mathrm{b}, \mathrm{i}} / \Omega$ will be either 1 or 0 , depending on which boundaries we choose to rotate. However, we will concentrate on the symmetrically driven cavity ( $\$ 4) \Omega_{\mathrm{t}, \mathrm{b}, \mathrm{i}} / \Omega=1$ and make some limited comments regarding the other case $\Omega_{\mathrm{b}} / \Omega=1$ and $\Omega_{\mathrm{t}, \mathrm{b}} / \Omega=0$ in our concluding discussion (§5).

We note that the special case of no inner cylinder can be addressed by setting $\eta=$ 0 , choosing $\Omega R_{\mathrm{o}}$ as the velocity scale and applying boundary conditions appropriate for the axis of symmetry at $r=0$. This also leads to replacing (2.4) by $v=r \Omega_{\mathrm{t}, \mathrm{b}} / \Omega$.

\section{(a) Convergence and validation of numerical results}

Before initiating the laboratory experiments, a numerical code (formulated as described previously) was used to provide an overview of the parameter space and thereby identify parameter values of interest. Numerical calculations were performed using the primitive variable formulation of the incompressible NavierStokes equations. The algorithm employs a finite-element method, using quadrilateral elements with biquadratic interpolation of the velocities and discontinuous piecewise linear interpolation of the pressure. The system is formulated and solved using the ENTWIFE (Cliffe 1996) finite-element library.

To ensure accuracy of the numerical results, two checks were applied. First, we compute the flow for a rotating inner cylinder with the top/bottom boundary conditions replaced with the natural boundary condition for the swirl component $v$; this results in a solution with little axial variation, which can be compared to the well-known circular Couette exact solution. Data for the convergence of the numerical results in this special case are provided in table 1 . Our second check is to recompute the known results of Mullin et al. (2000) and also to compare (by removing the inner boundary condition and making the domain simply connected) with the experimental data of Escudier (1984), for which full agreement was found in both cases. 
Clearly, there is a discontinuity in the boundary conditions for $v$ in the corner regions where the rotating top/bottom end wall meets a stationary cylinder. In the experimental configuration, a small fluid-filled gap is present between such boundaries. To eliminate the corner discontinuity, and to better model the experimental configuration, we apply a continuous velocity distribution on the top/bottom end walls which varies rapidly from the appropriate linear profile to zero over a small distance near the corner. To be consistent with the interpolation scheme, we choose a quadratic profile for the smoothing over the corner element boundary. Furthermore, we apply a corner refinement of the finite-element mesh to resolve this rapid variation and verify that the numerical results are insensitive to both the length scale over which the smoothing of the discontinuity is achieved (for sufficiently small length scales) and the inclusion of further refinement to the mesh.

All the computations presented herein are for a non-uniformly distributed mesh with $64 \times 48$ elements spread over the half-domain $(r, z) \in[0,1] \times[0,1 / 2]$, with symmetry conditions applied at $z=0$. Additional computations were performed over the full domain $(r, z) \in[0,1] \times[-1 / 2,1 / 2]$ for which no symmetry assumptions were made. We shall discuss the possibility of symmetry-broken flows in $§ 3$.

\section{Experimental investigation}

The experimental configuration consisted of a pair of concentric cylinders mounted in a controlled environment (a water jacket), through which a temperature-controlled fluid was pumped. This configuration ensured that the working section of the experiment was maintained at $25.0 \pm 0.02^{\circ} \mathrm{C}$. The outer cylinder of the flow domain was held stationary, while either the top/bottom end plates and the inner cylinder were rotated or just the bottom plate was rotated. The outer boundary was constructed from a precision ground glass cylinder with an inner diameter of $63.5 \pm 0.025 \mathrm{~mm}$. The inner cylinder was a $4.75 \mathrm{~mm}$ diameter rod located centrally on the axis of rotation.

In terms of our non-dimensional parameters, this geometry corresponds to a radius ratio of $\eta=0.0748$. The bottom and top end plates had a diameter of $63.48 \mathrm{~mm}$, thus leaving a small gap between the driving plates and the outer boundary. The distance between the upper and lower plates was chosen to give an aspect ratio of $\Gamma=0.78$ in the experiments presented herein.

A stepper motor with a gear box and toothed belt drive was used to drive the rotation of the boundaries. The angular frequency of the rotation was determined accurately from the input to the stepper motor, and we could therefore estimate the Reynolds number given the above length scales and the viscosity of the working fluid. The fluid was chosen to be a water-glycerol mixture $(50: 50$ by volume) and the viscosities were determined using an Ubbelohde viscometer.

The flow field was visualized by adding a small quantity of Mearlmaid AA natural pearlessence and illuminating the experiment from the side by a sheet of light. Photographs were taken from a position perpendicular to the plane of illumination using a digital camera, or a film camera where very long exposures were required. 
To generate the flow states visualized in the experimental pictures, we smoothly varied the angular frequency of the driving boundaries from rest to a final speed in small increments.

\section{Results: symmetrically driven flows}

We begin by computing the parameter space boundaries at which the first recirculation bubble appears in the meridional plane. The parameter space is spanned by the aspect ratio $I$, the Reynolds number $R e$ and the radius ratio $\eta$. Given these three parameters, we shall choose to present results by fixing the radius ratio $\eta$, and then describe the two-dimensional parameter space spanned by $\Gamma$ and $R e$.

The previous work of Mullin et al. presented results relevant to this case, showing that, for low values of $\eta$, there is a Reynolds number above which an obvious recirculation bubble can be observed (for the whole range of aspect ratios). On increasing the radius ratio $\eta$, the boundary for the appearance of this first bubble moves more rapidly to higher values of $R e$. Thus, for $\eta \geq 0.5$, it is difficult to obtain reliable numerical results for the boundary above which this flow feature can be observed because large values of $R e$ are required. It is therefore unclear whether the flow feature is absent above a critical value of $\eta$ or whether this is merely a rapid limiting process as $\eta \rightarrow 1$.

As has been noted by Tsiverblit (1993), the appearance of the recirculation bubble is not related to an instability of the flow and therefore it is difficult to provide a definite criterion for its presence. This is particularly the case if the bubble appears or disappears near a boundary, since we recognize that recirculating eddies can always occur when sufficiently close to such corner regions; these local eddy cascades were described in the work of Moffatt (1964) with a rotational analogue described by Hills (2001). The approach we take here is one that was successfully employed by Mullin et al. (1998); the criterion for the existence of a bubble is the presence of a recognizably enclosed region in the meridional streamline plot when viewed over the full computational domain.

In the two cases of $\eta=0.1$ and 0.2 , the range of existence is shown in figure 2 . Here we see a new feature that is absent in the less resolved data of Mullin et al. (1998), namely the presence of two limit points in the dataset. The 'kink' highlighted in the data of figure 2 has some physical significance. On the righthand branch, the recirculation bubble appears first through the creation of stagnation points ${ }^{2}$ at the inner cylindrical boundary. However, on the left-hand branch, the recirculation bubble appears through the creation of stagnation points in the bulk flow, along the plane of symmetry (i.e. on $z=0$ ).

We note that the kink in the datasets of figure 2 is a consequence of the presence of an inner cylinder. When the inner cylinder is absent, there is no such feature, as shown by the results of Mullin et al. (1998). Nevertheless, the feature is robust and the results are not qualitatively altered by a change in the radius ratio from $\eta=0.1$ to 0.2 , as demonstrated in figure 2 .

At aspect ratios between the two limit points shown in figure 2, on increasing the Reynolds number from zero, the recirculation bubble appears first at the inner cylinder, but on increasing the Reynolds number further, the bubble

${ }^{2}$ Here we are referring to stagnation points in the meridional cross section; clearly, there is still a swirl component to the velocity field at these locations. 


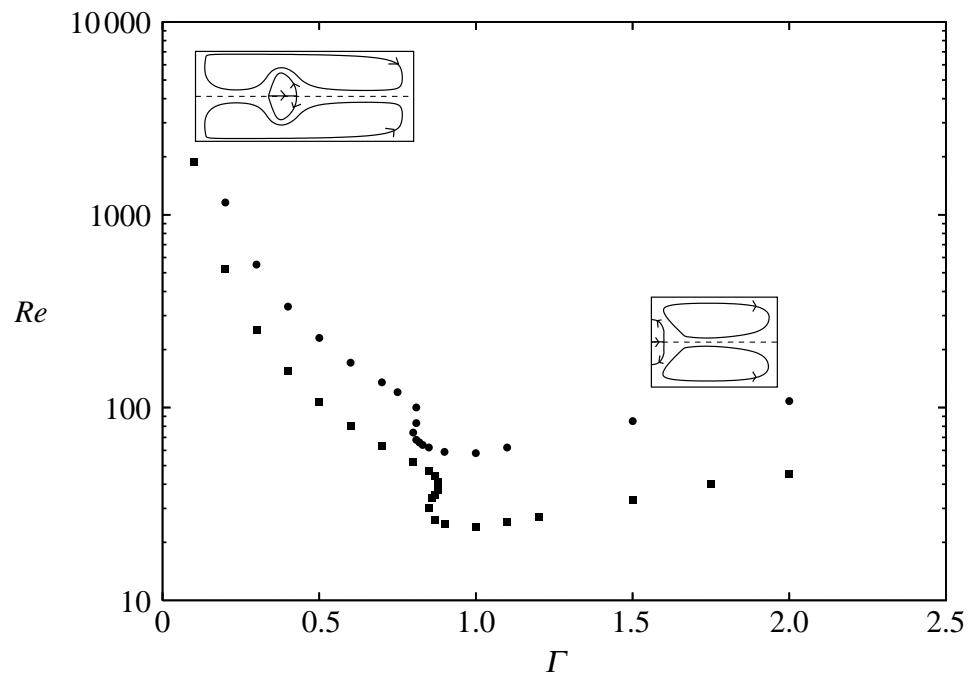

Figure 2. Data for the boundary above which at least one recirculation bubble exists. The lower dataset is for a radius ratio of $\eta=0.1$ and the upper dataset is for $\eta=0.2$. The inset meridional streamline plots show (schematically) the location of the recirculation bubble on crossing the existence boundary for low values (left) and high values (right) of $\Gamma$.

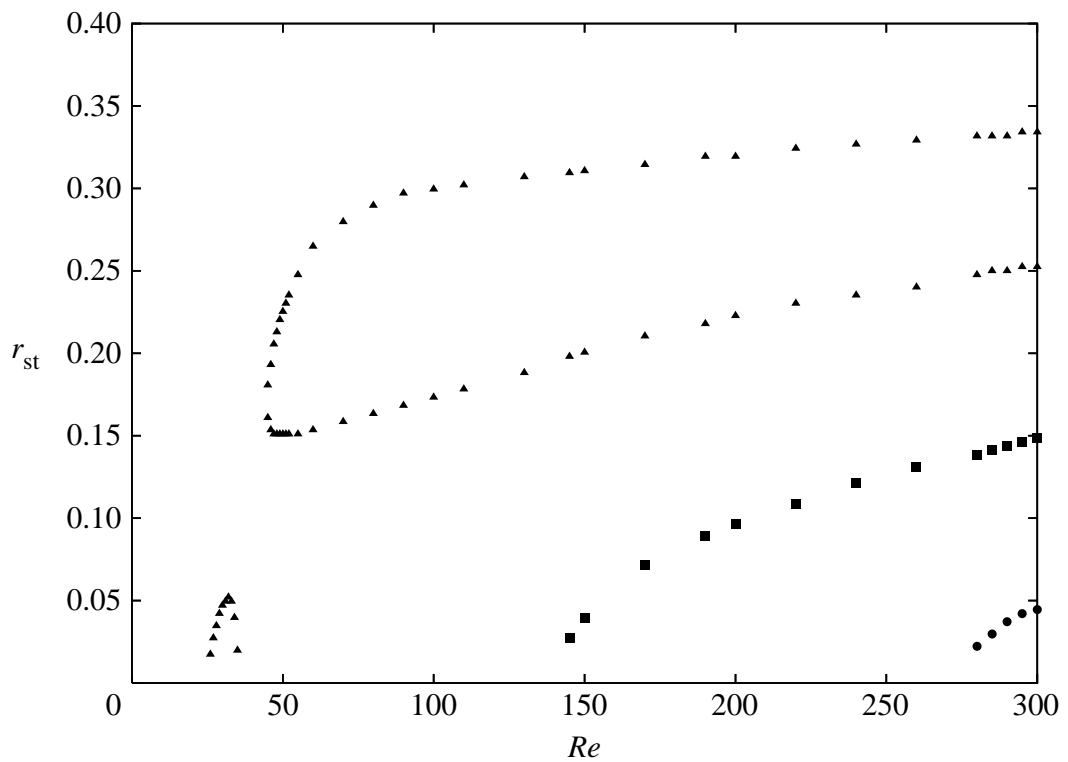

Figure 3. Data for the radial location of the stagnation points, $r_{\mathrm{st}}$, on the symmetry mid-plane $(z=0): r=0$ is the inner boundary and $r=1$ is the outer boundary. These data are for the case $\Gamma=0.865$ and $\eta=0.1$.

disappears (again at the inner cylinder) before reappearing in the bulk flow as a detached toroidal vortex. In figure 3 , we measure the radial position (in terms of the dimensionless coordinate $r \in[0,1]$ ) of the stagnation points as they appear on the mid-plane of symmetry, $z=0$, for increasing Reynolds number when $\Gamma=0.865$ 
(a)

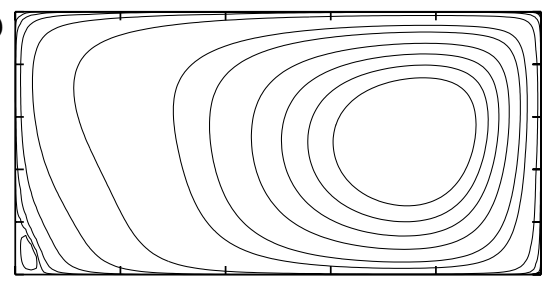

(b)

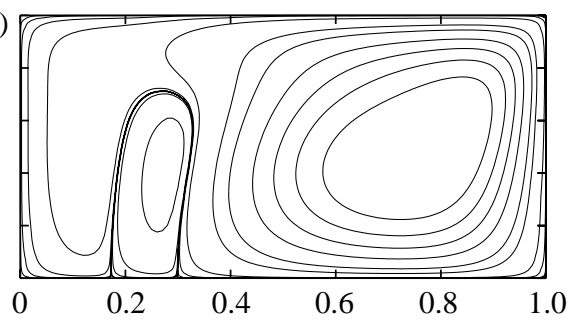

(c)

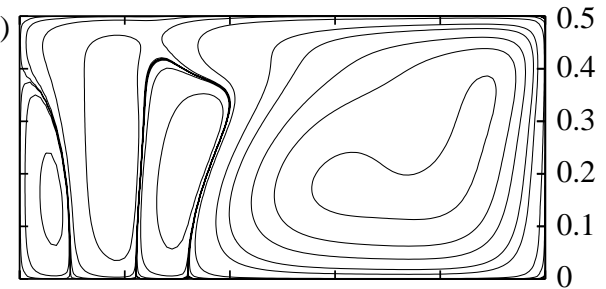

(d)

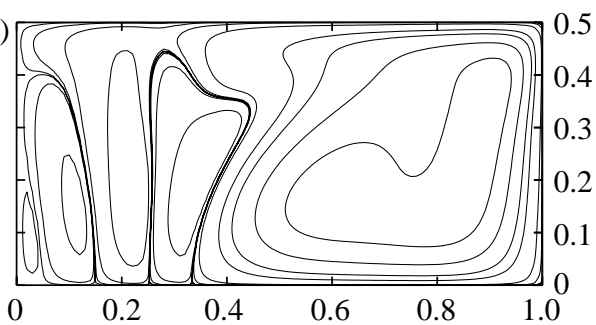

Figure 4. Predicted streamline patterns in the meridional $(r, z)$ plane for $\Gamma=0.865, \eta=0.1$ (as in figure 3) and $R e=30,100,200,300$ corresponding to $(a-d)$, respectively. Note that each image is only one-half $(z \in[0,1 / 2])$ of the full symmetric flow pattern that exists for $z \in[-1 / 2,1 / 2]$.

and $\eta=0.1$. This value for the aspect ratio is chosen to lie between the two limit points shown in the lower data of figure 2 . As can be observed from figure 3 , the first recirculation bubble appears $(R e \approx 26)$ and disappears $(R e \approx 35)$ at the inner boundary $r=0$, before occurring again $(R e \approx 45)$ in the main bulk of the flow as described above. In addition, figure 3 also shows that the detached bubble continues to exist for increasing $R e$ and that additional bubbles then occur at the inner cylinder (at $R e \approx 145$ and 280).

In figure $4 a-d$, we show examples of the meridional streamlines at a range of Reynolds numbers for the parameters corresponding to figure 3 . In these streamline images, it is clear that there is a cascade of recirculation zones that preserve the reflectional symmetry of the flow.

To assess the stability of these steady flow states, we sought bifurcations to states with broken (mid-plane) reflectional symmetry; however, none were found in the parameter ranges described herein. We must conclude that, in the absence of an instability leading to time dependence and/or loss of axisymmetry, these new states should be experimentally observable.

Given this prediction of stability, we seek these free-standing toroidal vortex states in the laboratory by using the experimental configuration outlined in $§ 3$. A flow visualization image of the meridional plane is shown in figure $5 a$ for parameter values above the first existence boundary, showing the presence of a single recirculation bubble. Since the aspect ratio $\Gamma=0.78$ is below the kink in the existence curve (figure 2), the bubble appears in the main bulk flow rather than at the inner cylinder. Figure $5 c$ also shows a corresponding numerical computation for the meridional streamlines and we see that there is an excellent agreement for the flow structure. As far as the authors are aware, this is the first experimental confirmation that these flow states are stable and observable. We emphasize that the bubble observed in figure $5 a, b$ develops suddenly at the central position shown, rather than occurring first at the inner boundary and subsequently migrating into the bulk flow. 
(a)

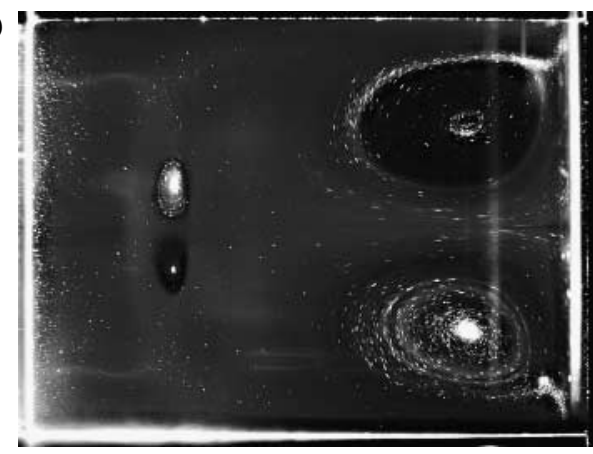

(c)

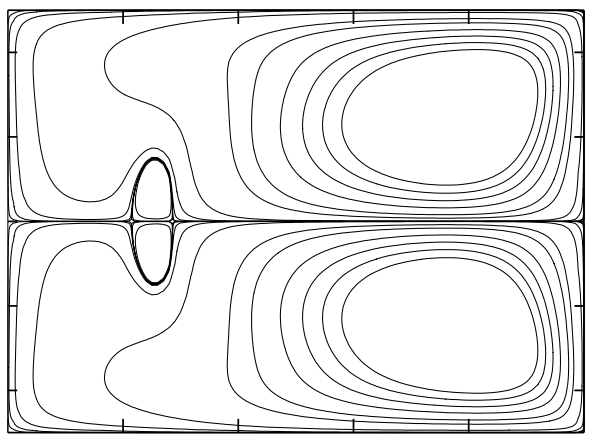

(b)

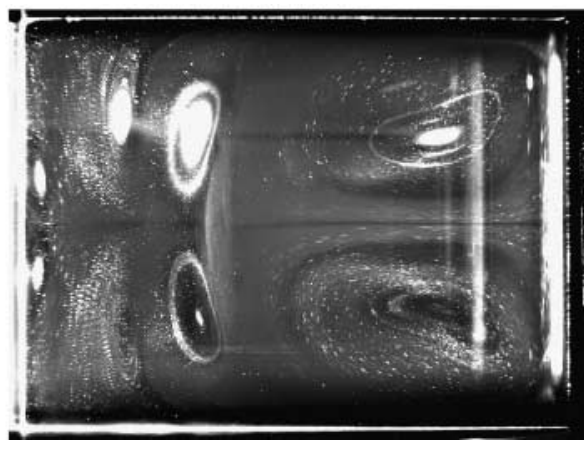

(d)

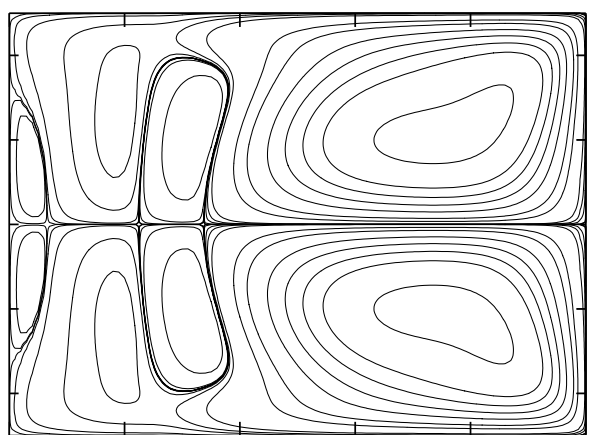

Figure 5. $(a, b)$ Flow visualization of the laboratory experiment and $(c, d)$ the corresponding numerical computation of the meridional streamlines for $\Gamma=0.78, \eta=0.0748$ and $(a, c) R e=45$ and $(b, d) R e=100$.

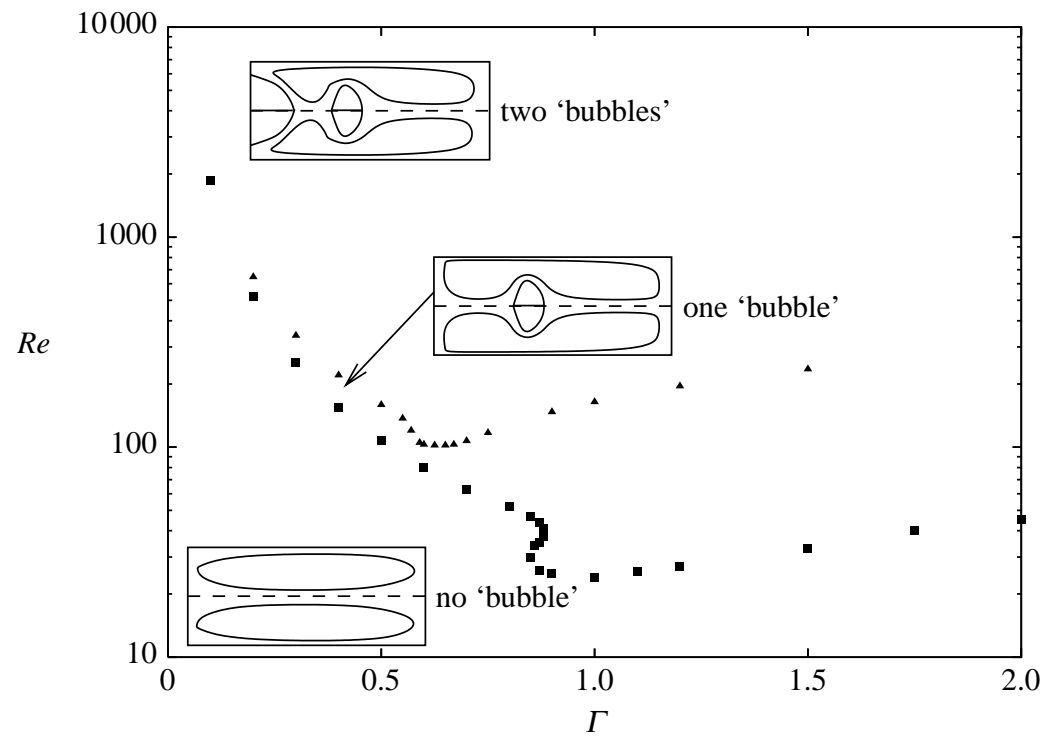

Figure 6. Boundaries above which the first (lower) and second (upper) recirculation 'bubbles' exist for $\eta=0.1$. The inset schematics show the qualitative structure for low aspect ratio in the three regions of the parameter plane. Additional boundaries exist (at larger $R e$ ) above which the flow field includes more recirculation regions. 
In figure $5 b, d$, we maintain the same aspect ratio and radius ratio (namely $\Gamma=0.78$ and $\eta=0.0748$ ) but continue to increase the rotation frequency of the boundaries up to $R e=100$. At this Reynolds number, we see that an additional stagnation point/line has developed at the mid-plane $z=0$, appearing first at the inner boundary and developing outwards to reveal an additional bubble to the left, adjacent to the inner cylinder.

Given that a second bubble clearly exists, we can identify the boundary in the $R e-\Gamma$ plane above which this feature is found (for a fixed radius ratio). In figure 6 , we repeat the data for the existence of the first bubble for $\eta=0.1$ (as shown in figure 2) and add to this the boundary for existence of a second bubble. Numerical evidence leads us to conjecture that a cascade of such boundaries exists, each of which corresponds to the inclusion of additional bubbles into the flow field. However, we should note that, in the laboratory experiments, the flow eventually exhibits what appears to be an instability to a travelling wave through a Hopf bifurcation as the rotation rate is increased.

\section{Discussion}

We have shown that toroidal vortices are a generic feature of swirl-driven cavity flows. In the symmetrically driven cavity, we observe a sequence of well-defined Reynolds numbers (at fixed values of the radius ratio, $\eta$, and aspect ratio, $\Gamma$ ) at which closed recirculation zones appear in the meridional flow field (the so-called 'bubbles'). The appearance of these bubbles has been associated by several authors with the phenomenon of vortex breakdown, as a steady analogue of that behaviour. While this interpretation remains controversial, the mechanism behind the sudden development of the bubble in these highly controlled flows remains a largely unanswered question. There is undoubtedly a rapid development of the bubble beyond the existence boundaries shown in figure 6 , and evidence of a square-root growth in the dimensions of the bubble (figure 7). In this sense, it is not surprising that the flow feature was mistakenly viewed as an instability in early work.

If any progress is to be made theoretically with regard to describing the origin of these toroidal vortices, it is clear that the simplest case would be the limit of an asymptotically small aspect ratio. In this limit, one might expect the flow to be a rigid-body rotation over the majority of the flow domain, with some transitional region near the outer cylinder. Questions then arise concerning the location and appearance of the sequence of bubbles. It is interesting in this regard to note that the scaling of the existence boundaries shown in figure 6 is such that

$$
R e=\frac{c}{\Gamma^{2}}+\frac{\alpha}{\Gamma}+\cdots
$$

as $\Gamma \rightarrow 0$. In figure 8, we repeat the data for the existence of the first and second bubbles, but rescale the Reynolds number, instead using $\overline{R e}=\operatorname{Re} \Gamma^{2}(1-\eta) / \eta$. Additionally, figure 8 fits a straight line through the available data for the range $\Gamma<0.5$. Moreover, figure 8 provides strong evidence that the constant $c$ is the same for the first and second bubbles; indeed, we conjecture that any subsequent sequence of existence boundaries would scale with the same manner. One might interpret this scaling as showing that the development of consecutive bubbles is a 


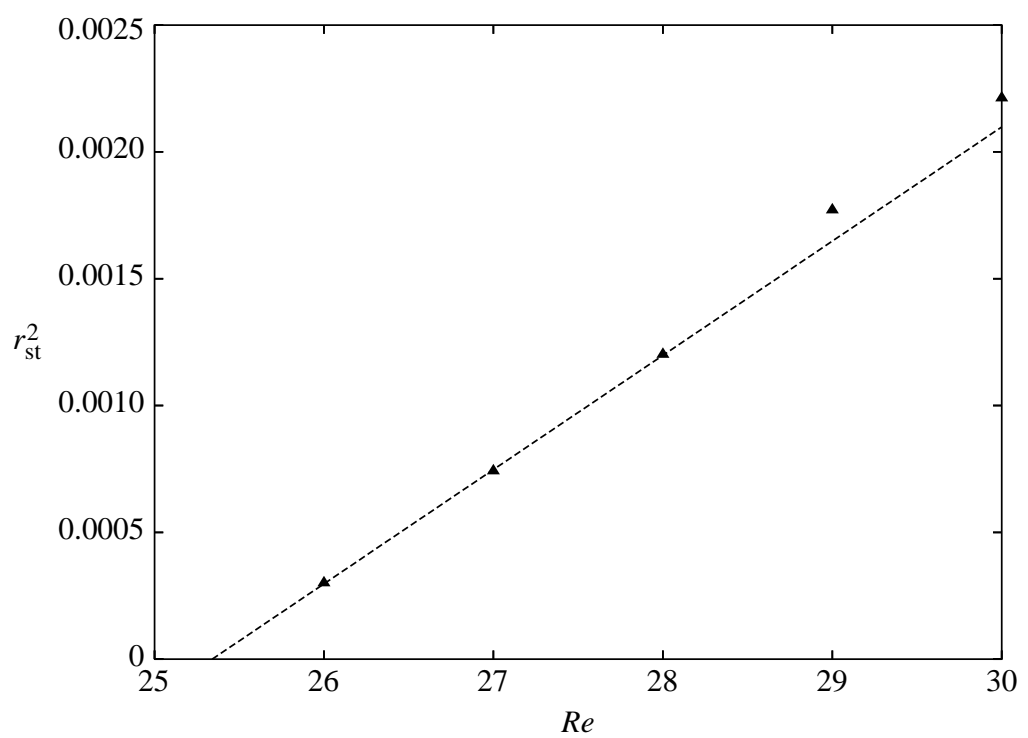

Figure 7. The radial location of the first appearing stagnation point, as denoted by $r_{\text {st }}$, for $\Gamma=0.865$ and $\eta=0.1$ (figure 3 ). Here we show the evidence of the square-root growth in the bubble size at the onset by plotting $r_{\text {st }}^{2}$ together with a linear fit near the onset.

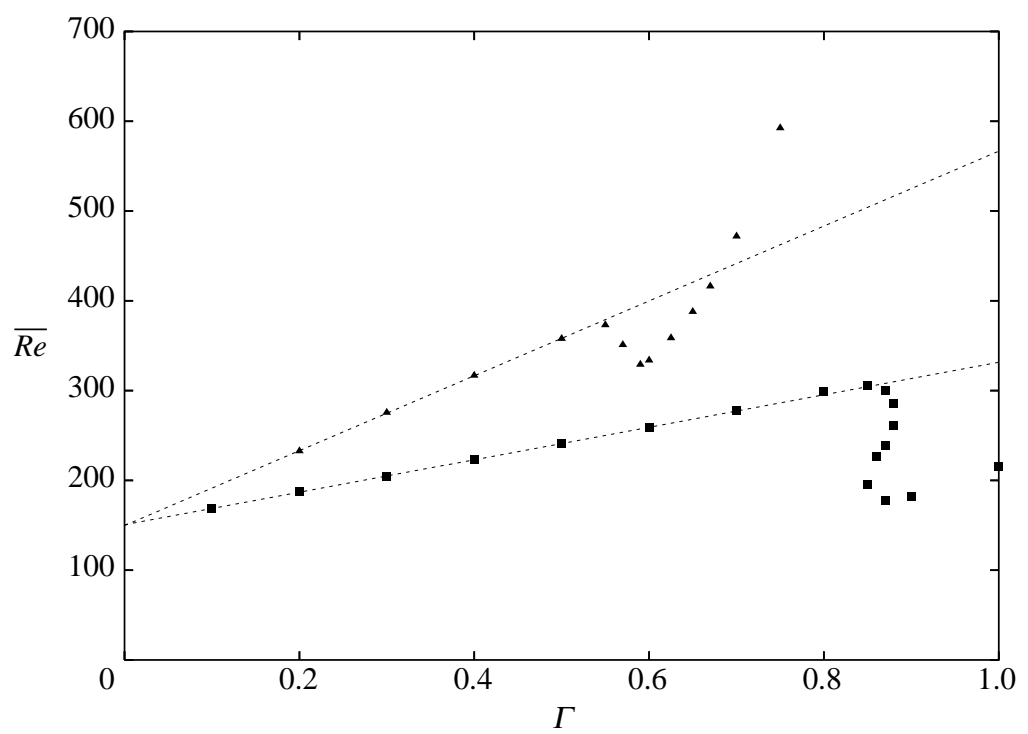

Figure 8. The onset of the first and second 'bubbles' in the limit of a small aspect ratio for $\eta=0.1$. Here $\overline{R e}$ is the rescaled Reynolds number $\overline{R e}=\operatorname{Re} \Gamma^{2}(1-\eta) / \eta=\Omega H^{2} / \nu$. The lower dataset (squares) is the appearance of the first bubble and the upper dataset (triangles) is the second bubble.

manifestation of the same effect. Furthermore, the quantity $\overline{R e}=\Omega H^{2} / \nu \approx 150$ is clearly of some significance in (axially) short cavities. An asymptotic examination of this problem may reveal more details regarding the factors that determine the rapid bubble development. 


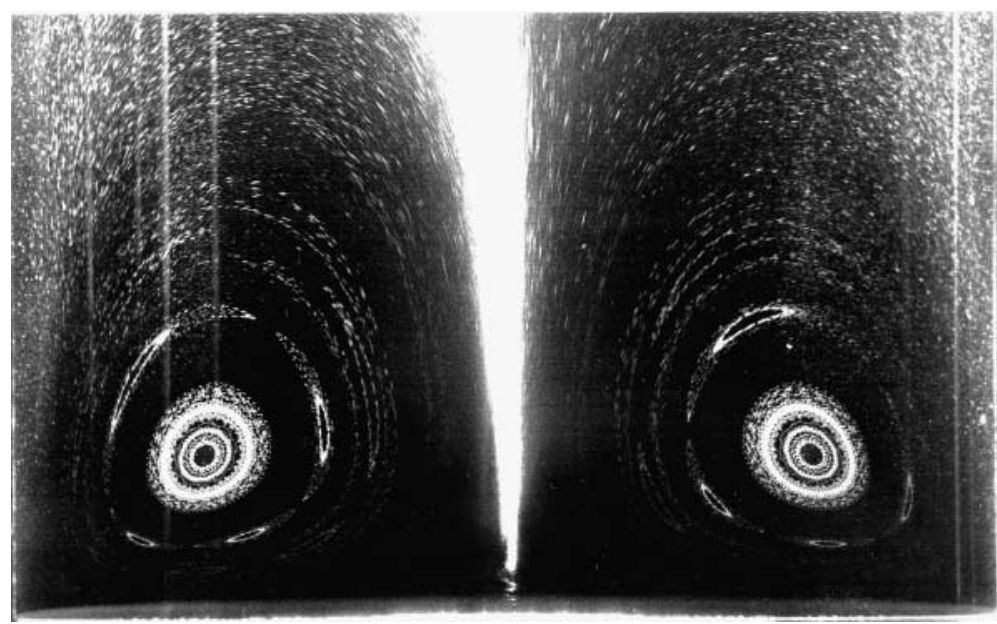

Figure 9. Non-axisymmetric particle paths shown by a light sheet across the full diameter of the cylinder (the overexposed central region is the axis). In this case, only the lower boundary (shown) is rotating, with $\Gamma=3$ (the full height of the domain is not shown), $\eta=0$ (there is no inner boundary) and a Reynolds number of $\Omega_{\mathrm{b}} R_{\mathrm{o}}^{2} / \nu=30$ (the absence of an inner cylinder in this case requires this alternative definition). The exposure time for the photograph is approximately $330 \mathrm{~min}$.

During the course of this investigation, we also briefly examined toroidal eddy states driven by the rotation of a single end wall. In these cases, Hills (2001) demonstrated that a cascade of eddies can be obtained in the low Reynolds number limit for large aspect ratios. These states are somewhat different from those described in the previous sections of this work, being an axisymmetric analogue of the eddy structure described by Moffatt (1964). The vortices arise because the axial decay of an induced azimuthal flow is sufficiently rapid (spatially) for the far-field response in the meridional flow to be dominated by a spatially oscillatory (axial) eigenmode.

In a cylindrical geometry, this eigenfunction-dominated structure takes the form of a sequence of stacked toroidal vortices that fill the full radial extent of the domain and have a well-defined axial wavelength. Nevertheless, as with the classical corner vortices described by Moffatt, the observation of this structure is made challenging by the rapid geometric reduction in the vortex strengths as one moves away from the driving boundary. Indeed, in this geometry, the decay rate is significantly greater than the two-dimensional problem of Moffatt, leading to each vortex being approximately 14000 times weaker than the preceding one in the limit of $R e \rightarrow 0$ and in the absence of an inner cylinder.

Attempts to visualize this type of weak vortex flow over long time scales lead to some interesting observations. In figure 9, we show an image of the first (strongest) toroidal vortex (using the same method that proved successful in figure 5). In this case, a much longer exposure time of approximately $330 \mathrm{~min}$ was used. Every effort was made to ensure that the flow domain remained axisymmetric, that the lid of the driving boundary was perpendicular to the side walls and that the axis of rotation of the driving boundary was centrally located. No measurable evidence of a deviation from axisymmetry has been obtained in our experimental apparatus in the absence of flow seeding. 
It seems that these standard flow visualization techniques must be interpreted with regard to the non-Lagrangian nature of the seeding particles. Although over small exposure times the deviation from being a perfect Lagrangian tracer of the underlying flow is not significant, over the larger time scales required to capture weak vortex motion, the presence of non-axisymmetric particle paths is clearly visible. There are obvious similarities between this image and those obtained by (for example) Fountain et al. (2000), showing chaotic advection in similar flows that are forced to have a significant non-axisymmetry by a sloped rotating wall (visualized by dye).

The authors acknowledge financial support from EPSRC (grant GR/R72983/01). The work of T.M. is also supported by an EPSRC Senior Fellowship. Contributions from Serco and the advice of K. A. Cliffe enabled the authors to obtain results using the ENTWIFE numerical library for nonlinear elliptic PDEs.

\section{References}

Cliffe, K. A. 1996 ENTWIFE (release 6.3) reference manual. AEAT-0823. Oxford, UK: Harwell Laboratory.

Escudier, M. P. 1984 Observations of the flow produced by a rotating end wall. Exp. Fluids 2, 189-196. (doi:10.1007/BF00571864)

Fountain, G. O., Khakhar, D. V., Mezic, I. \& Ottino, J. M. 2000 Chaotic mixing in a bounded three-dimensional flow. J. Fluid Mech. 417, 265-301. (doi:10.1017/S002211200000118X)

Hills, C. P. 2001 Eddies induced in cylindrical containers by a rotating end wall. Phys. Fluids 13, 2279-2286. (doi:10.1063/1.1384470)

Moffatt, H. K. 1964 Viscous and resistive eddies near a sharp corner. J. Fluid Mech. 18, 1-18. (doi:10.1017/S0022112064000015)

Mullin, T., Tavener, S. J. \& Cliffe, K. A. 1998 On the creation of stagnation points in a rotating flow. J. Fluid Eng. 120, 685-689.

Mullin, T., Kobine, J. J., Tavener, S. J. \& Cliffe, K. A. 2000 On the creation of stagnation points near straight and sloped walls. Phys. Fluids 12, 425-431. (doi:10.1063/1.870320)

Tsiverblit, N. 1993 Vortex breakdown in a cylindrical container in the light of continuation of a steady solution. Fluid Dyn. Res. 11, 19-35. (doi:10.1016/0169-5983(93)90003-S)

Valentine, D. T. \& Jahnke, C. C. 1994 Flows induced in a cylinder with both end walls rotating. Phys. Fluids 6, 2702-2710. (doi:10.1063/1.868159)

Vogel, H. U. 1968 Experimentelle Ergebnisse ber die laminare Stromung in einem zylindrischen Gehause mit darin rotierender Scheibe. Phys. Fluids 6, 2702. 